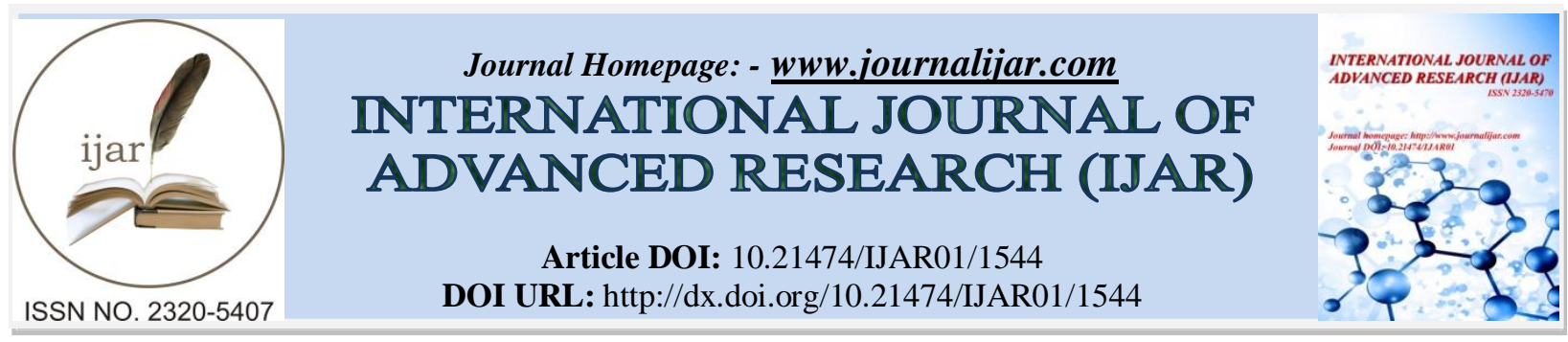

RESEARCH ARTICLE

\title{
HYBRID FIREFLY SWARM INTELLIGENCE BASED FEATURE SELECTION FOR MEDICAL DATA CLASSIFICATION AND SEGMENTATION IN SVD - NSCT DOMAIN.
}

\author{
B.Thamaraichelvi* and G.Yamuna. \\ Department of Electrical Engineering, Annamalai University, Annamalai nagar, Tamil Nadu, India.
}

\section{Manuscript Info}

Manuscript History

Received: 16 July 2016

Final Accepted: 23 August 2016

Published: September 2016

Key words:-

Fire Fly (FF), Particle Swarm

Optimization (PSO), Singular Value

Decomposition (SVD), Gray Level Co-

Occurrence Matrix (GLCM), Non-Sub

Sampled Contourlet Transform (NSCT),

Support Vector Machine (SVM)

Classification.

\section{Abstract}

In this paper, the diagnosis of childhood Atypical Teratoid /Rhabdoid tumor (AT/RT) in magnetic resonance brain images and Hemochromatosis in Computed Tomography (CT) liver images, through the hybridization of particle swarm optimization and firefly (PSO-FF) algorithms for feature selection has been presented. Here, the features are extracted through Non-sub Sampled Contourlet Transform (NSCT) to collect the information in all the directions including the edges from the images, Singular Value Decomposition (SVD) to enhance the image and to get the algebraic details, Gray Level Co-occurrence Matrix (GLCM) method to obtain the statistical textural features from the images. All these features are fused together, then the hybridized meta-heuristics algorithms are applied to extract the salient features from the feature set. The ability of global thinking ( $\mathrm{g}_{\text {best }}$ ) in PSO has been combined with the local search capability of firefly to achieve good results. The Radial Basis Function - Support Vector Machine (RBF-SVM) classifier has been used for the classification of brain and liver disease. The affected part was segmented by using expectation maximization algorithm.

Copy Right, IJAR, 2016,. All rights reserved.

\section{Introduction:-}

In central nervous system the Atypical Teratoid/Rhabdoid tumor is a serious disease in which the cancer cells form inside the tissues of brain of a child in the age of three. Since, it is fast growing, it must be diagnosed in the early stage, ,if not which may affect the survival of the child soon. Mostly, these tumors form in the cerebellum or brain stem. The cerebellum is the part of the brain which controls movement, balance and posture. Loss of balance, Nausea, headache and increase in head size of infants are the symptoms of this disease.

Hemochromatosis is an iron overload disorder caused by the progressive increase in iron storage and deposition in some body organs particularly with liver. The diagnosis becomes evident in the middle age (30-40), but in the case of women the clinical manifestation may be delayed till the post- menopausal period. Hence, the proposed system deals with the diagnosis of these pathological disorders with the survey of the following literatures.

Abdenour and Mekhmoukh proposed a new segmentation method based on an improved fuzzy c-means clustering with particle swarm optimization (pso) technique to select the cluster centre combined with level set method for outlier rejection [1]. Balasubramanian vijayalakshmi and vasudev mohan presented a kernel based PSO algorithm for feature selection to detect a plant leaf type based on texture, shape and color charecteristics, finally, the Fuzzy 
Relevance Vector Machine (FRVM) is employed to characterize the type of leaves[2]. Esmael Hamuda et al. reviewed the challenges and limitations of current segmentation methods based on colour index, threshold and learning approaches with advantages and disadvantages[3]. A.N. Benaichouche et al. Implemented a new algorithm using pso initialization of fuzzy c-means with Mahalanobis distance to reduce the influence of the geometrical shape of the different classes and reallocating the misclassified pixels [4]. Yong Zhang et al. Studied a method to overcome the problem of relative membership of fuzzy c-means with Possibilistic $c$-means algorithm and replacing the Euclidean distance with mahalanobis distance by optimizing the initial cluster centre using particle swarm optimization technique [5].

Devraj Mandal et al. Introduced a new algorithm to solve for energy minimization problems in active contour models with particle swarm optimization technique which can be used for both scalar and vector valued images [6]. Pablo Mesejo et al. surveyed the most notable and recent works on metaheuristics based hybrid segmentation approach with deformable models, helps in parameter selection and initial boundary location [7]. Jothi G. and Hannah Inbarani H. Made a hybridization of two techniques, Tolerance Rough Set (TRS) and Firefly Algorithm (FA) to select the features, for MRI brain image segmentation and tumor detection.[8] . V. Rajinikanth, and M.S. Couceiro developed an optimal multi-level image segmentation using the Firefly Algorithm (FA) [9]. Adán JoséGarcía, and Wilfrido Gómez-Flores analysed nearly Sixty-five clustering methods based on nature-inspired meta heuristics and formulated the challenges for automatic clustering models [10]. Satyasai Jagannath Nanda and Ganapati Panda reviewed all major nature inspired metaheuristic algorithms employed for partitional clustering including the major area of application. [11]. Xin-She Yang explained well about firefly algorithm and reviewed the variants, together with a selection of parameters[12]. Iztok Fister et al. carried out a comprehensive review in Swarm Intelligence to show that the firefly algorithm could be applied to every problem arising in practice [13].

Nguyen Cong Long et al. integrated a rough sets based attribute reduction using chaos firefly algorithm and interval type-2 fuzzy logic system to handle with high-dimensional dataset for the diagnosis of heart disease [14]. Mohammed Alweshah and Salwani Abdullah hybridized the firefly algorithm with simulated annealing, where simulated annealing is applied to control the randomness step inside the firefly algorithm, while optimizing the weights of the standard PNN model to improve the performance of the PNN [15]. Yong Zhang et al. applied a feature selection method based on bare bones particle swarm optimization for solving classification problems. Here, a reinforced memory strategy is designed to update the local leaders of particles for avoiding the degradation of outstanding genes in the particles, and a uniform combination is proposed to balance the local exploitation and the global exploration of algorithm [16]. Sandeep Chaplot et al. employed a novel method using wavelets as input to self organising map neural network and support vector machine (svm) thereby achieving good classification rate for svm[17]. Fahd M.A. Mohsen et al. implemented a new multilevel thresholding segmentation method based on particle swarm optimization (pso). Here, pso is used to find the best values of thresholds for the appropriate partition of image according to the fitness function [18].

H.Hannah Inbarani et al. developed a hybrid feature selection method based on particle swarm optimization and rough sets for medical diagnosis[19]. Bae- Muu Chang et al. implemented a novel classification method using support vector machine, which extracts rotation invariant image texture features in singular value decomposition and discrete wavelet transform domains [20]. Nelly Gordillo et al. gave an overview of the most relevant brain tumor segmentation methods including advantages and disadvantages, which makes the researchers to choose the best method easily for their work [21]. Amita Kumar and Rajesh Mehra et al. developed a hybrid approach for classification of brain tissue in MRI based on Particle Swarm Optimization (PSO) and Support Vector Machine (SVM). Here, wavelet based texture feature are extracted from normal and tumor region by using HAAR wavelet and these features are given as input to the SVM classifier [22]. S. Anu H. Nair' and P.Aruna analysed a method to fuse different biometric modalities such as the Discrete Cosine Transform (DCT), Singular Value Decomposition (SVD) and Bacterial Foraging Optimization Algorithm (BFOA) . It is found that the watermarked images are found robust over different attacks and they are able to reverse the biometric template for Bacterial Foraging Optimization Algorithm (BFOA) watermarking technique [23].

The rest of the paper is organized as follows. Section 2 reviews the methodology of the proposed work. Section 3 presents the methods used for feature extraction. Section 4 deals with the techniques applied for feature selection. Segmentation and classification methods are discussed in section 5. Section 6 interprets the results obtained with each of the selected methods. Finally, the last section presents the conclusion and potential future work. 


\section{Methodology:-}

The input magnetic resonance brain images and computed tomography liver images are pre-processed using weighted median filter and the features are extracted through Non-sub Sampled Contourlet Transform (NSCT), Singular Value Decomposition (SVD) and Gray Level Co-occurrence Matrix (GLCM) techniques and fused together, then hybrid particle swarm optimization and firefly method is used to select the most efficient features so as to achieve high segmentation precision and classification performance to diagnose the disease properly. While, GLCM is more effective in describing the visual features and SVD in describing the internal features. NSCT is mainly used with spherical and circular shaped images thereby extracting the rotation invariant image features.

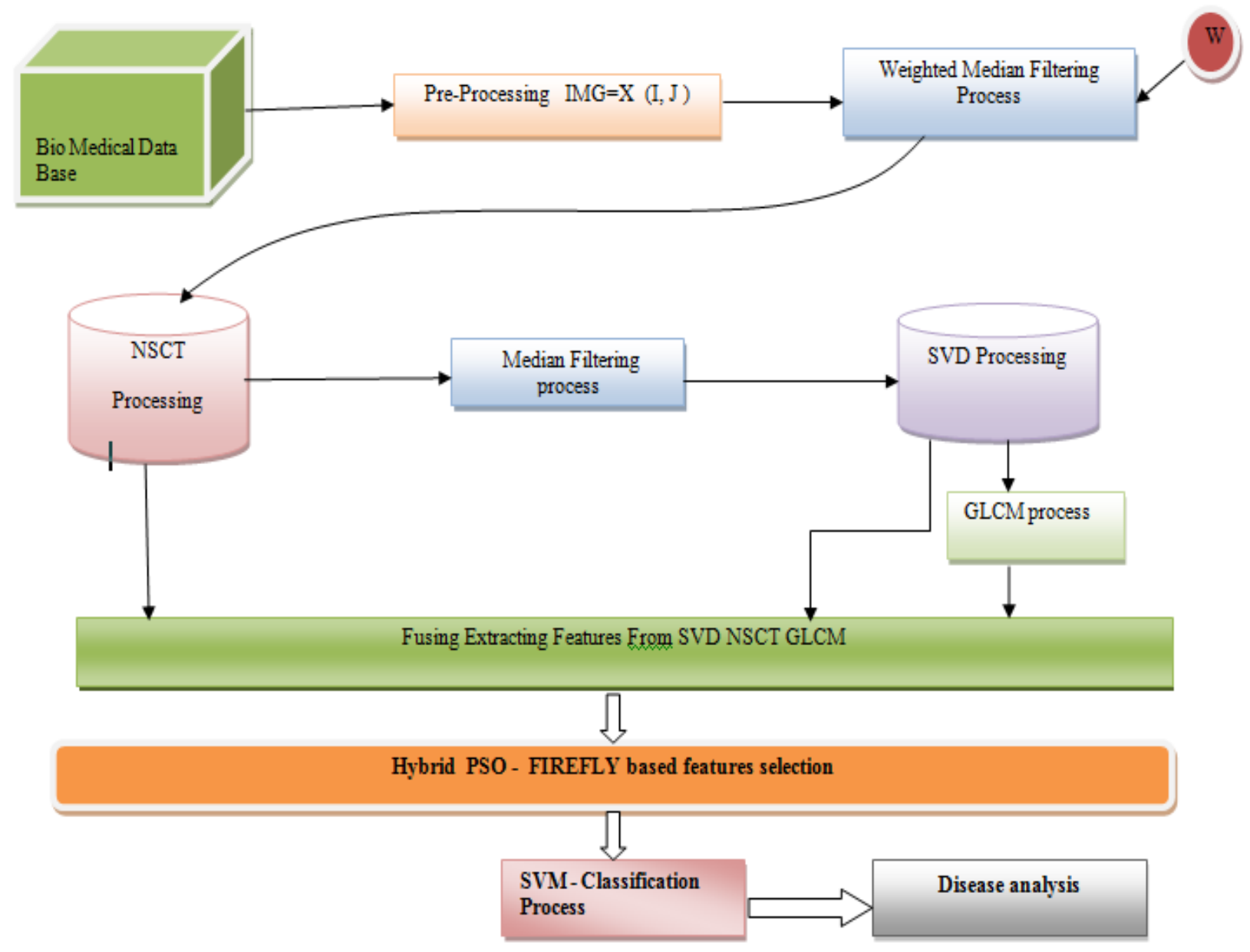

\section{Feature extraction:-}

Figure 1:- Graphical representation of proposed methodology.

The features must be extracted from the region of interest to analyse the characteristics of the image depending upon the requirement. In our proposed work, the features are extracted through the following techniques.

\section{Contourlet transform:-}

Due to the limitation in separability only a horizontal, vertical, and 45 degree component can be easily recognized by 2-D wavelet transform. Eventhough the edges can be analysed the directional information about the edges cannot be obtained. Contourlet transform is the discrete extension of the curvelet transform mainly applied to capture curves instead of points. It is an edge detection algorithm which combines the multiscale and the directional decomposition of images used mainly with a high curve content. In the contourlet structure double filter bank is used to obtain the smooth contours of images. First, the laplacian pyramid structure (LPS) is used to capture the point of discontinuities. Secondly, the directional filter bank (DFB) is applied to form those dis-continuities into linear structures [24]. Contourlet transform in general is not shift invariant. 


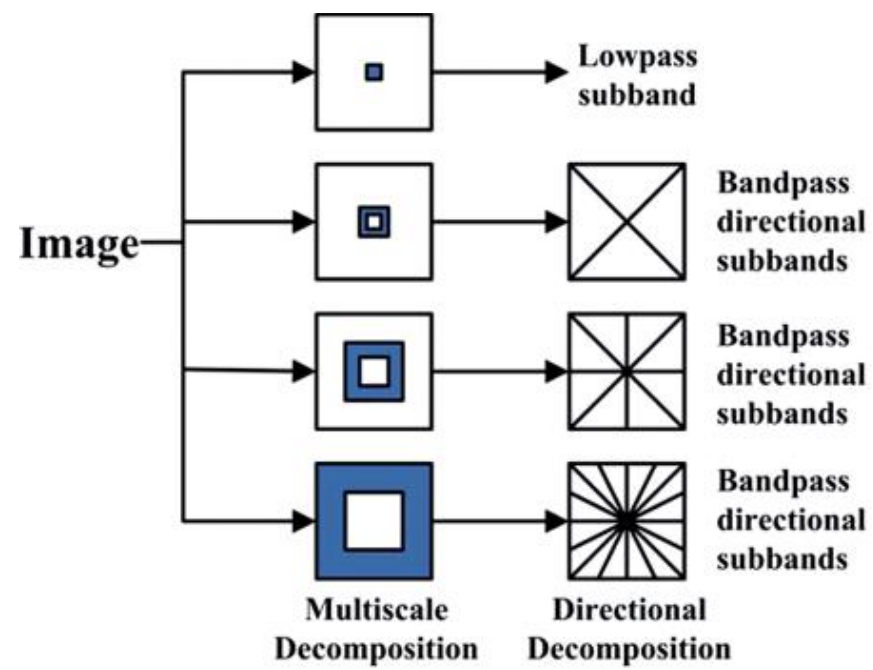

(a)

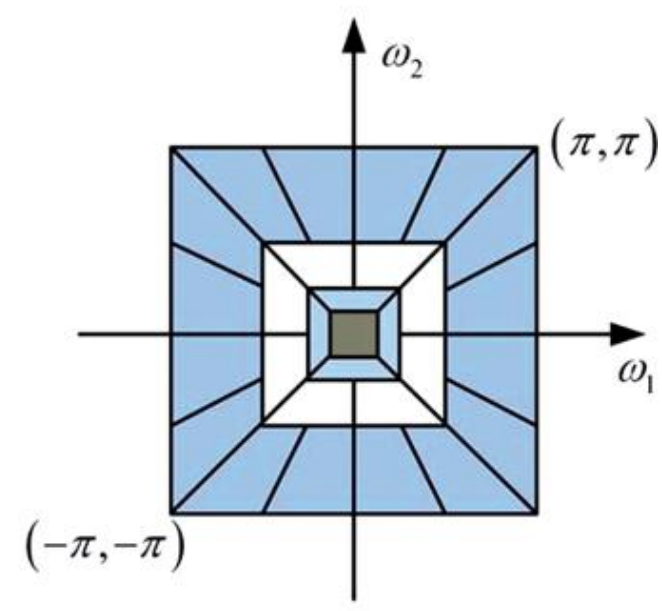

(b)

Figure 2:- Filter bank for contourlet transform.

\section{Non-Sub Sampled Contourlet Transform (NSCT) :-}

To overcome the drawback of contourlet transform Non-sub Sampled Contourlet Transform (NSCT) has been introduced. The non-sub sampled contourlet transform was developed by Cunha, Zhou \& Do in 2006. The laplacian pyramid in contourlet was replaced by a non-sub sampled pyramid structure to retain the multi scale properties of the images. The non-sub sampled directional filters are also used to capture the directional information. A flexible multiscale, multidirectional and shift invariant image decomposition can be efficiently implemented with the help of NSCT. The structure of NSCT is divided into Non- sub Sampled Pyramid Filter Bank (NSPFB) and Non- sub Sampled Directional Filter Bank (NSDFB). In this transform, the multi scale analysis has been made first on the images followed by multi - directional analysis $[25,26]$. The decomposition process can be explained as follows.

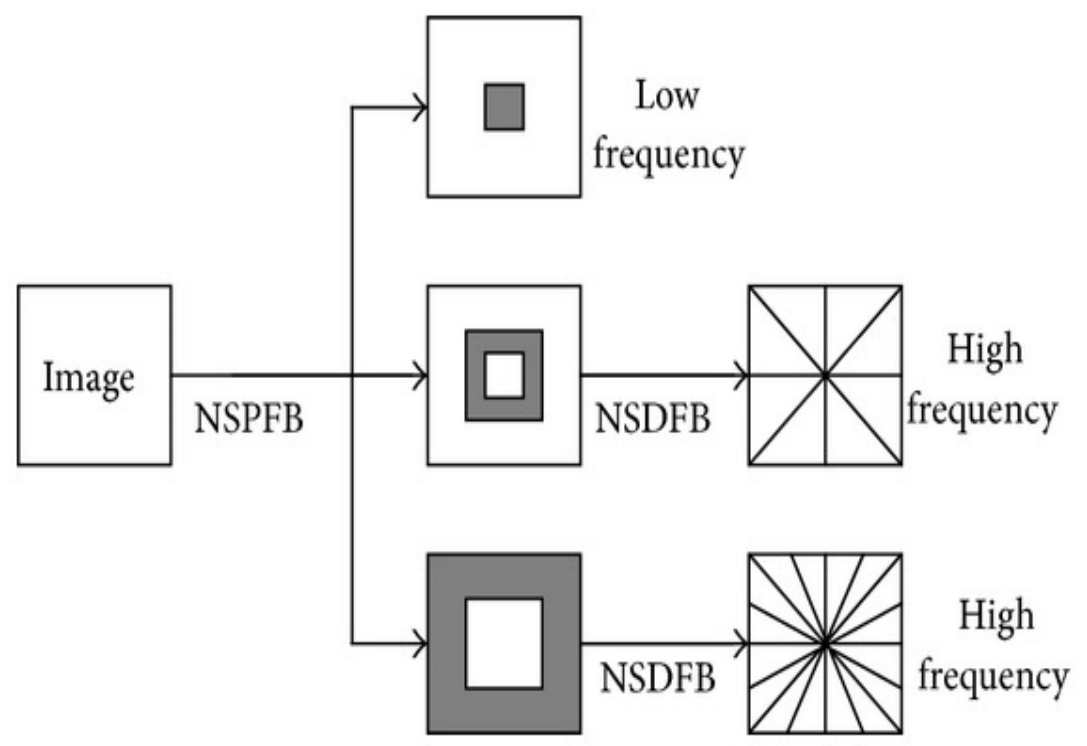

Figure 3:- Decomposition of image using NSCT.

In the first stage, the image is decomposed to have one low pass sub-band image and different high pass sub-band images. Since, there is no down-sampling all the sub-band images have the same sizes with the original images. In the second stage, the multi directional decomposition is applied to the various high frequency sub- band images, to retain the sub-band coefficients at different directions. so as to obtain a series of multi-resolution, multi-direction and translation invariant frequency domain sub-images. 


\section{Singular Value Decomposition (SVD):-}

The Singular Value Decomposition (SVD) is an important tool in signal processing and statistical data analysis. It provides a new way for extracting algebraic features from an image. Singular value decomposition is a technique used to extract rotation-invariant textural features from the image $[20,27]$. The singular value decomposition of a matrix A of the image I with size ' $\mathrm{MxN}$ ' is the factorization of A into the product of three matrices, $\mathrm{A}=\mathrm{UDV}^{\mathrm{T}}$ where the columns of $\mathrm{U}$ and $\mathrm{V}$ are orthonormal and the matrix $\mathrm{D}$ is diagonal with positive real entries. $\mathrm{A}=\mathrm{UDV}^{\mathrm{T}}$ Where $\mathrm{U}(m \times m)$ and $V(n \times n)$ are orthogonal matrix,. The columns of the orthogonal matrices $U$ and $V$ are called the left and right singular vectors respectively [29]. An important property of $U$ and $V$ is that they are mutually orthogonal.

The singular value decomposition can be computed using the following observations:-

- The left-singular vectors of $\mathrm{D}$ are a set of orthonormal eigenvectors of $\mathbf{A A}^{*}$.

- The right-singular vectors of $\mathrm{D}$ are a set of orthonormal eigenvectors of $\mathbf{A}^{*} \mathbf{A}$.

- The non-zero singular values of D (found on the diagonal entries of $\mathrm{D}$ ) are the square roots of the non-zero eigenvalues of both $\mathbf{A}^{*} \mathrm{~A}$ and $\mathbf{A} \mathbf{A}^{*}$.

\section{Gray Level Co-Occurrence Matrix (GLCM):-}

Gray level co-occurrence matrix (GLCM) is recognized as an important tool for textural analysis, which shows the spatial relationship between a pixel (one with gray level $i$ and the other with gray level $j$ ) with its immediate neighbouring pixel separated by a distance $d$ in a given direction $\theta$. It calculates how often pairs of pixel with specific values and in a specified spatial relationship occur in an image, thereby creating a gray level co-occurrence matrix from which the statistical measures can be extracted [28-30]. In the actual texture analysis, the gray level cooccurrence matrix often is not used directly, while the second degree statistics are extracted on the basis of this matrix and used as the feature values of texture identification.

Hence, GLCM is included in the second order statistical texture analysis. It can only extract the textures under single scale and single direction. The use of glcm in wavelet domain ignores to retain the information in detail sub-band. To overcome the limitation of wavelet domain glcm, A kind of texture feature extraction method combining singular value decomposition and glcm is proposed, so as to achieve the extraction of texture features under multi-scale and multi-direction. A co-occurrence matrix ' $\mathrm{C}$ ' of an ' $\mathrm{n} \times \mathrm{m}$ ' image I, parameterized by an offset $(\boldsymbol{\Delta x}, \Delta \mathbf{y})$ is given as,

$$
C_{\Delta x, \Delta y}(i, j)=\sum_{p=1}^{n} \sum_{q=1}^{m} \begin{cases}1, & \text { if } I(p, q)=i \text { and } I(p+\Delta x, q+\Delta y)=j \\ 0, & \text { otherwise }\end{cases}
$$

where $i$ and $j$ are the intensity values of the image I, $p$ and $q$ are the spatial positions in the image I and the offset ( $\Delta x, \Delta y$ ) depends on the direction $\theta$ used and the distance $d$ at which the matrix is computed . Features generated using this technique are usually called Haralick features.

\section{Feature selection:-}

The intention of feature selection approach is to select a small subset of features which minimizes redundancy and maximizes relevance to the target such as the class labels in classification. Hence, feature selection plays a significant role in machine learning by providing high predictive accuracy using the selected prominent features.

\section{Particle Swarm Optimization (PSO) Algorithm:-}

Particle swarm optimization is a computational method mainly used to optimize a problem iteratively to get a solution with the given measure of quality $[16,19]$. It solves a problem by having a population of candidate solutions, and moving these particles around in the search-space according to simple mathematical formulae over the particle's position and velocity [20]. Each particle's movement is influenced by its local best known position but, is also guided towards the best known positions in the search-space, which are updated as better positions are found by other particles[22]. This is expected to move the swarm toward the best solutions. Meanwhile, the particle swarm optimization (PSO) algorithm is exploited to select a nearly optimal combination of features and a set of parameters utilized in the SVM. 
The mathematical model is given by,

$$
{ }_{v} v_{i}^{k+1}=w v_{i}^{k}+c_{1} \operatorname{rand}(\ldots) X\left(p_{\text {best }}-s_{i}^{k}\right)+c_{2} \operatorname{rand}(. .) X\left(g_{\text {best }}-s_{i}^{k}\right)
$$

Where,

$v_{i}^{k}$ - Velocity of the agent ' $\mathrm{i}$ ' at iteration ' $\mathrm{k}$ '

$\mathrm{w}$ - weighing function.

$c_{j}$ - weighing factor.

$s_{i}^{k}$ - Current position of agent ' $\mathrm{i}$ ' at iteration ' $\mathrm{k}$ '

rand - Uniformly distributed random numbers between 0 and 1 .

$p_{\text {best }}-p_{\text {best }}$ of agent (local best)

$g_{\text {best }}-g_{\text {best }}$ of the group.

$$
w=w_{\max -\left[\left(w_{\max }-w_{\min }\right) \text { Xiter }\right] / \max \text { iter }}
$$

Where,

$w_{\max }$ - initial weight.

$w_{\text {min }}$ - final weight.

Max iter - maximum number of iteration.

Iter - Current iteration number

$s_{i}^{k+1}=s_{i}^{k}+v_{i}^{k+1}$

The fitness function of this PSO method depends upon the error rate of the classifier. The error rate was calculated using the terms True Positive (TP), True Negative (TN), False Positive (FP) and False Negative (FN).

True Positive (TP) - Abnormal cases correctly classified.

True Negative (TN) - Normal cases correctly classified.

False Positive (FP) - Normal cases classified as abnormal.

False Negative (FN) - Abnormal cases classified as normal.

Error rate $=\frac{(F P+F N)}{(F P+F N+T P+T N)}$

The position of a particle represents the selected feature sub set. Hence, a new feature sub set has been created by avoiding the unselected features. The classifier performance is evaluated based on the new set of features. This procedure has been repeated with the updation of $p_{\text {best }}$ and $g_{\text {best }}$ values and this algorithm stops when the error rate is minimized.

\section{Fire Fly (FF) Algorithm:-}

The firefly algorithm is one of the important tool in swarm intelligence and utilised successfully in the area of optimization. It is a meta-heuristics algorithm and developed by Xin-She-Yang in the year 2008 [12]. It is inspired by the behaviour of fireflies. In general the fireflies produce a 'cold light' with no infrared or ultraviolet frequencies. Light intensity of each firefly is propotional to the quality of solution and each firefly needs to move towards the brighter fireflies. It depends on balancing between two major components: exploration and exploitation. Exploration is the process of discovering the diverse solutions within the search space. Exploitation means focusing the search process towards the best solution, thus exploiting the information found so far. 
The Assumptions are,

1. All the fireflies are unisex.

2. Brightness is determined by the objective function.

3. Attractiveness is propotional to brightness and inversely propotional to distance.

The movement of firefly ' $\mathrm{i}$ ' is attracted towards another more attractive (brighter) firefly ' $\mathrm{j}$ ' is determined as follows [13],

Movement:-

$$
x_{i}^{k+1}=x_{i}+\beta_{0} e^{-r_{i j}^{2}}\left(x_{j}-x_{i}\right)+\alpha(\operatorname{rand}-0.5)
$$

Where $\beta=0.2, \alpha=2$.

$\mathrm{r}-$ Distance between any two fire flies .

$\beta_{0-}$ Attractiveness at $\mathrm{r}=0$.

$\gamma$-Fixed light absorbtion co-efficient. ( $\gamma=0$, in absolute clear air, $\gamma=\infty$, in foggy air.)

Distance (r)

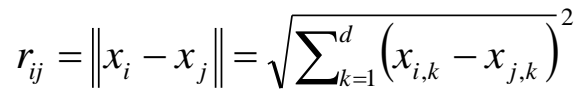

$\mathrm{d}-$ no. of dimensions

Fire fly algorithm often converge quickly.

\section{Proposed hybrid FF-PSO Algorithm:-}

Since, the firefly algorithm is oscillatory in nature, it cannot give the expected result under some circumstances. PSO algorithm depends upon the fitness function, where the parameters have to be tuned properly. Hence, the two meta-heuristics algorithms are hybridized to give proper result. The fireflies cannot give best solution faster in all occasions. Since, during clear air there is no light dispersion and the fire flies can see each other. But in foggy air, due to extreme light dispersion they cannot see each other, so, the balance between exploration and exploitation cannot be achieved properly. Due to this reason the firefly algorithm can be hybridized with other meta-heuristics algorithms. Hence, in this proposed method firefly has been combined with particle swarm optimization algorithm. Thereby, the ability of social thinking ( $\mathrm{g}_{\text {best }}$ ) in PSO has been combined with the local search capability of FF.

The proposed mathematical model for hybrid particle swarm optimization and firefly algorithm is given by,

$$
V_{i}^{k+1}=w V_{i}^{k}+\beta_{0} e^{-\gamma_{i j}^{2}}\left(p_{\text {best }}-x_{i}\right)+\alpha(\text { rand }-0.5)\left(g_{\text {best }}-x_{i}\right)
$$

The proposed algorithm is capable of selecting the features successfully from the fused feature set with minimum time.

\section{Segmentation and Classification:-}

Expectation maximization (EM) algorithm:-

Expectation maximization is a statistical method of segmentation used to produce good results especially with limited noise level. It is an efficient iterative process to compute the Maximum Likelihood (ML) estimates of model parameters when there is many-to- one mapping to the distribution governing the observation [31,32]. Each iteration of EM algorithm has two steps: The E-step and M-step. In E-step the missing data are estimated from the current estimates of the model parameters. In M-step, the likelihood function is maximized with the assumption that the missing data are known. The missing data, that are estimated from the E-step are used in case of actual missing data. Convergence is assured since the algorithm is guaranteed to increase the likelihood at each iteration.

\section{SVM Classifier:-}

The support vector machine (SVM) has higher classification accuracy than other classifiers with high-dimension feature vector. Also, the SVM serves as a multi classifier for image texture features. It is a linear machine developed from statistical learning theory and used in many fields like bio-informatics, image recognition, pattern classification 
etc. The objective of Support Vector Machine is to construct a hyperplane as the decision surface in such a way that the margin of separation between positive and negative support vectors is maximized [17,20]. In our proposed method Radial Basis Function (RBF) - SVM is used for classification process to identify whether the given image is normal or abnormal. RBF kernel maps the linear data space into non-linear feature space. This RBF utilizes the combination of supervised and unsupervised learning techniques. This approach can be used for modelling and classification. It can also be used for dimentionality reduction.

\section{Results and Discussion:-}

In this proposed method the meta-heuristics based feature extraction process has been handled with the merits of particle swarm optimization and firefly algorithms. The magnetic resonance brain images and the computed tomography liver images were collected from the Department of Radiology, Raja Muthaiya Medical College and Hospital (RMMCH), Annamalai University, and also from http//:www.radiopaedia.org. These images are preprocessed using weighted median filters with the window size of $3 \times 3$. The features are extracted through NSCT to collect the information from different directions including the edges. SVD method of extraction is used to enhance the image to collect the algebraic details from each and every sub bands. GLCM technique is combined with SVD to collect the statistical textural details from the sub bands. All the features extracted through different techniques are fused, then the hybridized PSO-FF meta-heuristics algorithm is used to select the salient features. The performance of PSO, FF and PSO-FF are analysed. These selected features are given to the RBF-SVM classifier to diagnose the Atypical Teratoid/ Rhabdoid tumor in magnetic resonance brain images and Hemocromatosis in CT liver images. Finally the affected area was segmented by applying Expectation maximization (EM) technique. The results are analysed as follows,

Figures 5,6,7 explain the interpretation of input features on the basis of time for PSO,FF, PSO-FF algorithms respectively. It can be proved that the hybrid PSO-FF method handles the set of incoming features with less time for the same no. of iterations, when compared with PSO and FF techniques.

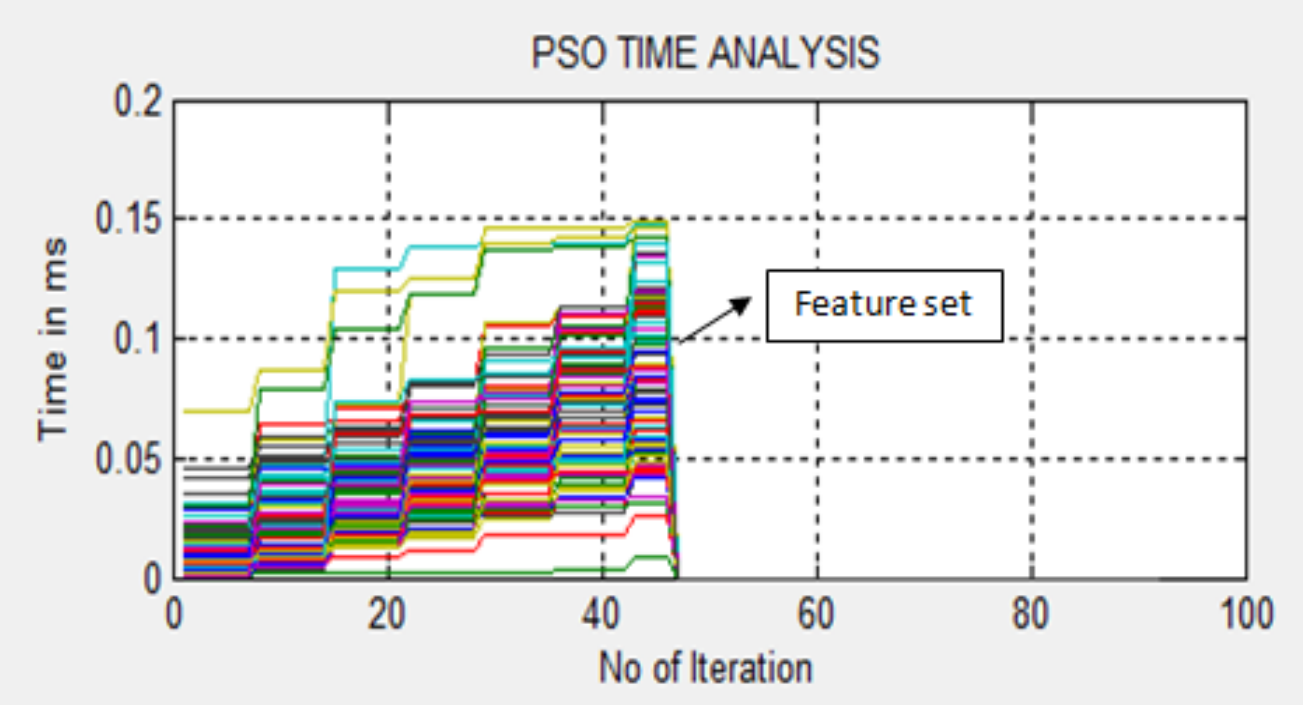

Figure 5:- Interpretation of set of input features by PSO technique. 


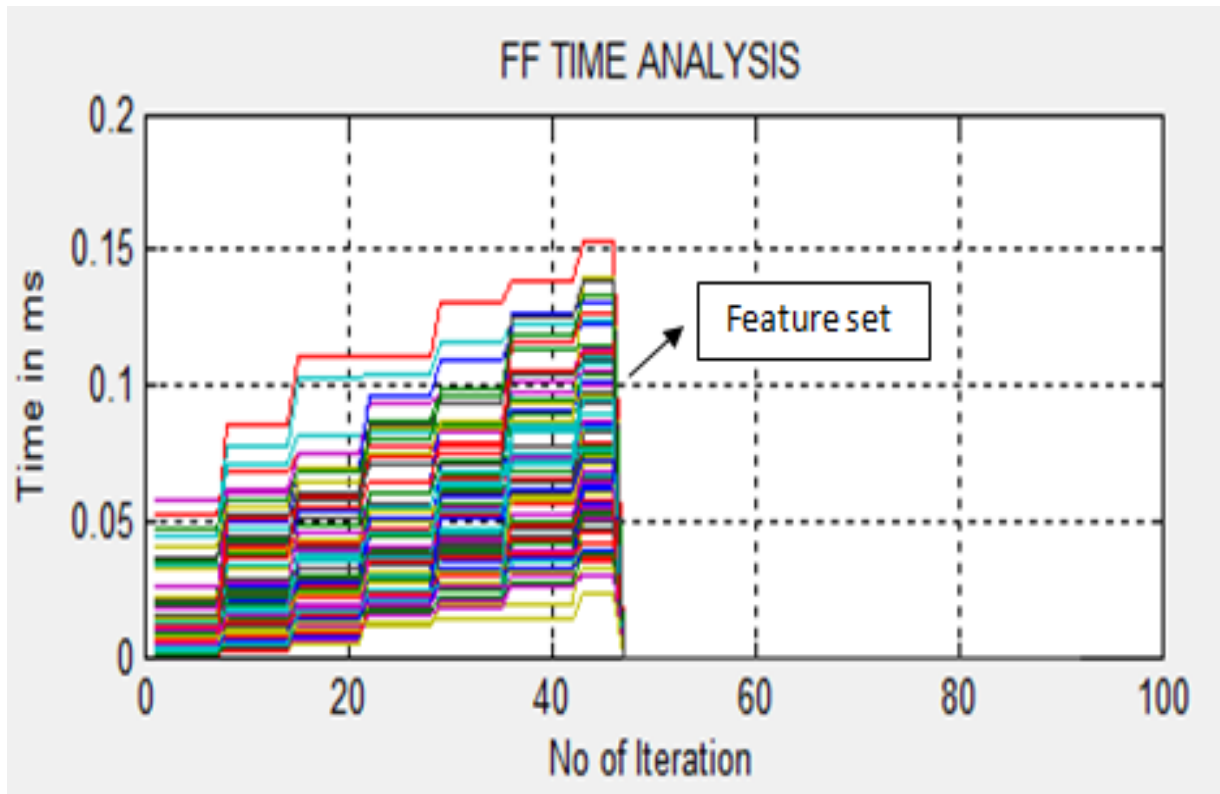

Figure 6:- Interpretation of set of input features by FF technique.

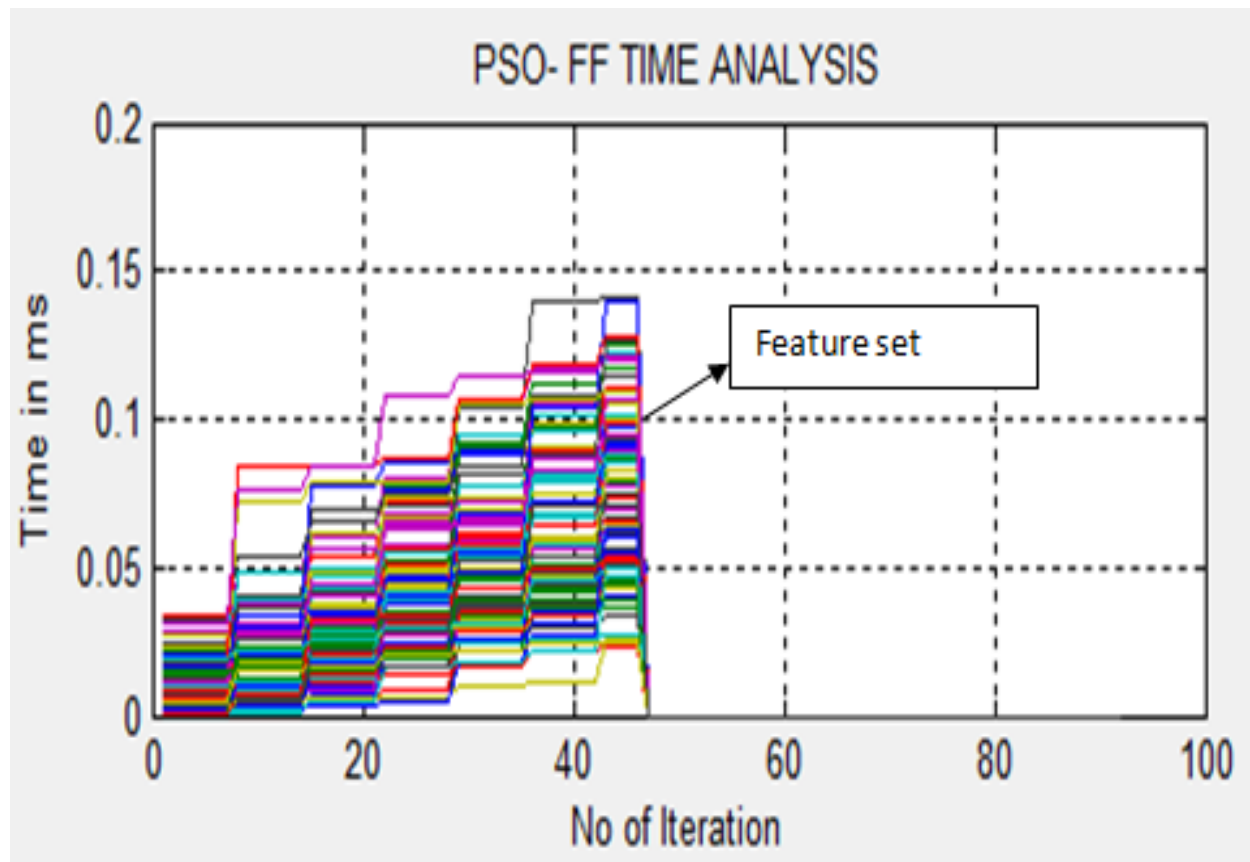

Figure 7:- Interpretation of set of input features by PSO - FF technique.

Figure 8 depicts the perfect diagnose of childhood Atypical teratoid/Rhabdoid tumor in magnetic resonance brain image. Table $1 \& 2$ show the details of feature extraction through SVD and glcm techniques. 


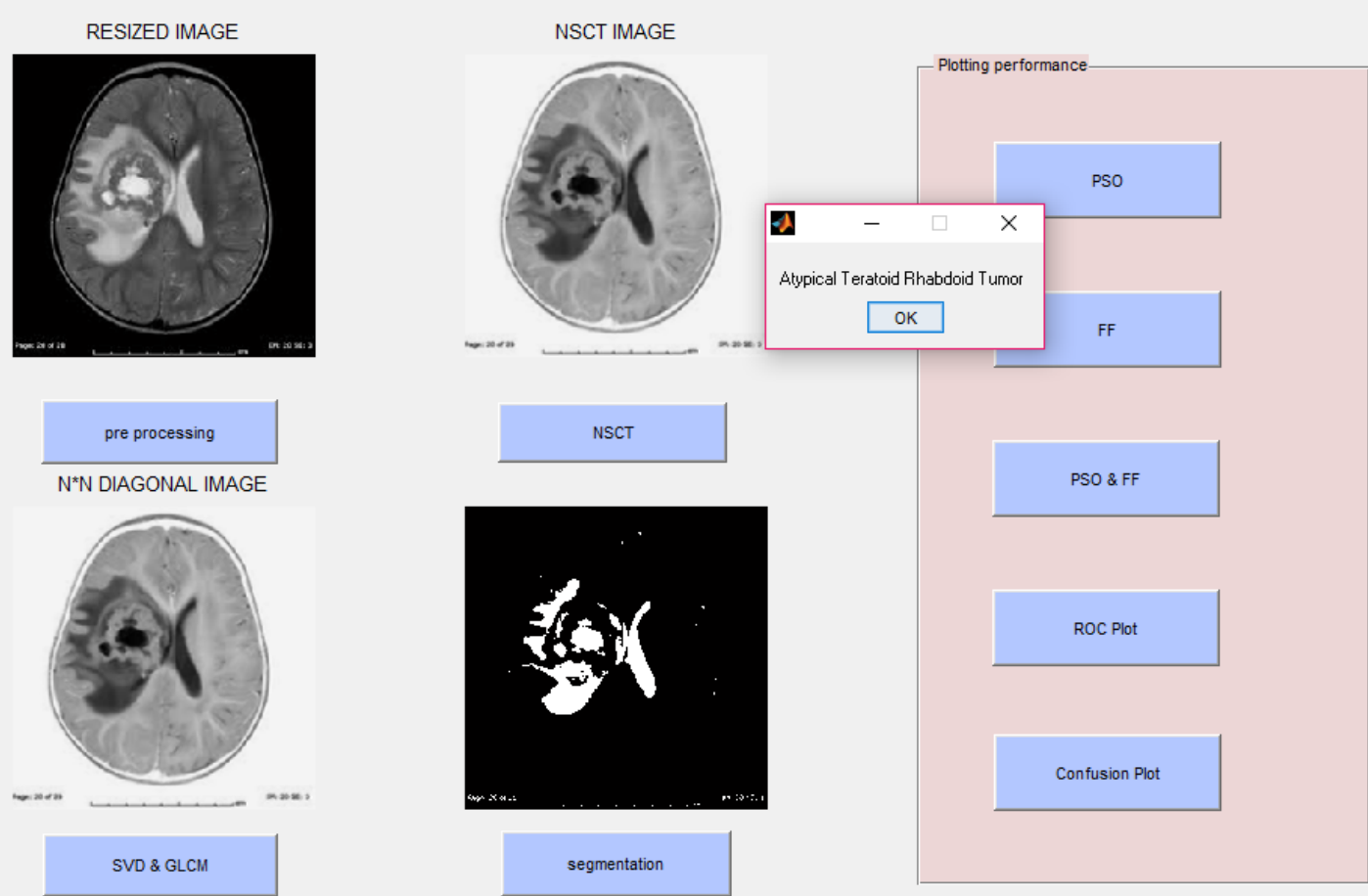

Figure 8:-. Perfect prediction of tumor in MRI brain image.

In table 1 the extracted feature values of brain MRI for left and right orthogonal matrices and the diagonal matrix through SVD are listed. Table 2 depicts the extracted features through GLCM for MRI brain. It has been found from table 2 that the correlation level and the homogeneity are around 0.9 , which is nearer to 1 as required. The contrast level and the energy level must be minimum that are around 0.25 .

Table 1:- Feature extraction through SVD method for mri brain image.

\begin{tabular}{|l|l|l|l|}
\hline SVD Features & 'U' matrix & Diagonal matrix $(\Sigma)$ & 'V' matrix \\
\hline Mean & 0.2602 & -269.7736 & 0.3404 \\
\hline Standard deviation & 0.0203 & 210.5647 & 0.3768 \\
\hline Min. Pix .Intensity & -0.0015 & -867.1333 & -0.0804 \\
\hline Max. Pix. Intensity & 0.0835 & 15.8333 & 1.6127 \\
\hline
\end{tabular}

Table 2:- Feature extraction through GLCM method for MRI brain image.

\begin{tabular}{|c|c|}
\hline GLCM Features & values \\
\hline Contrast & 0.2338 \\
\hline Correlation & 0.9771 \\
\hline Energy & 0.2374 \\
\hline Homogeneity & 0.9416 \\
\hline
\end{tabular}

Figures 9,10,11 describes about the range of features selected by PSO, FF, PSO-FF techniques individually. Fig.9 shows the feature selection range of PSO, the feature selection range is from 0- 0.06 which covers only 50\% .of pixels. 


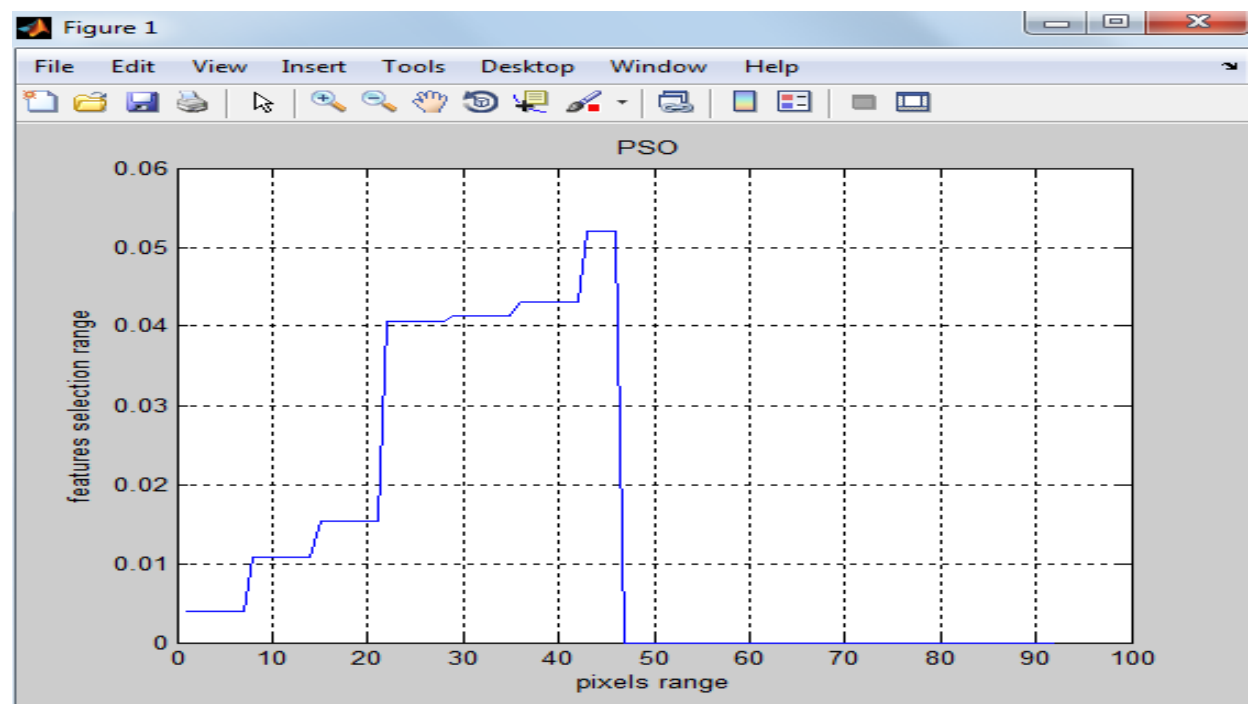

Figure 9:- The range of feature set selected by PSO technique to diagnose the brain disease.

Fig. 10 depicts the feature selection range of FF, the feature selection range is from $0-5 \times 10^{5}$ which covers only $10 \%$ of pixels due its fast convergence nature.

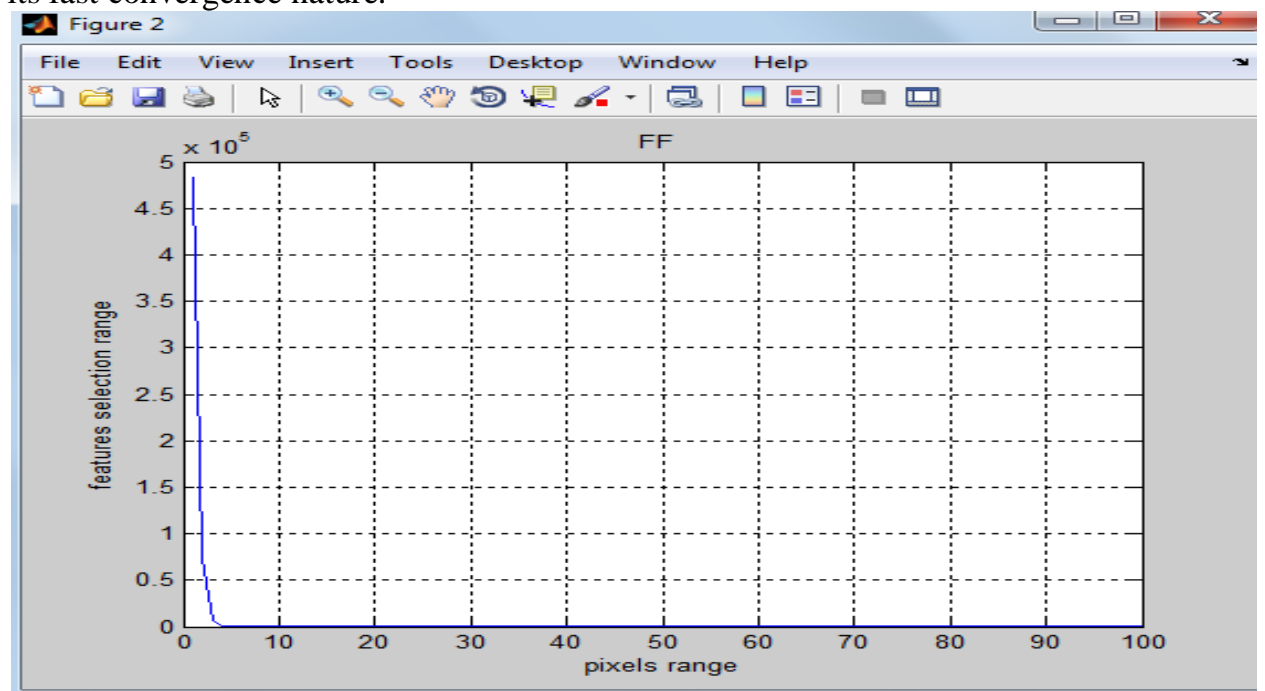

Figure 10:- The range of feature set selected by FF technique to diagnose the brain disease.

Fig. 11 shows the feature selection range of PSO - FF, the feature selection range is from 0- 2.56 which covers $75 \%$ of pixels as needed for the proposed method . 


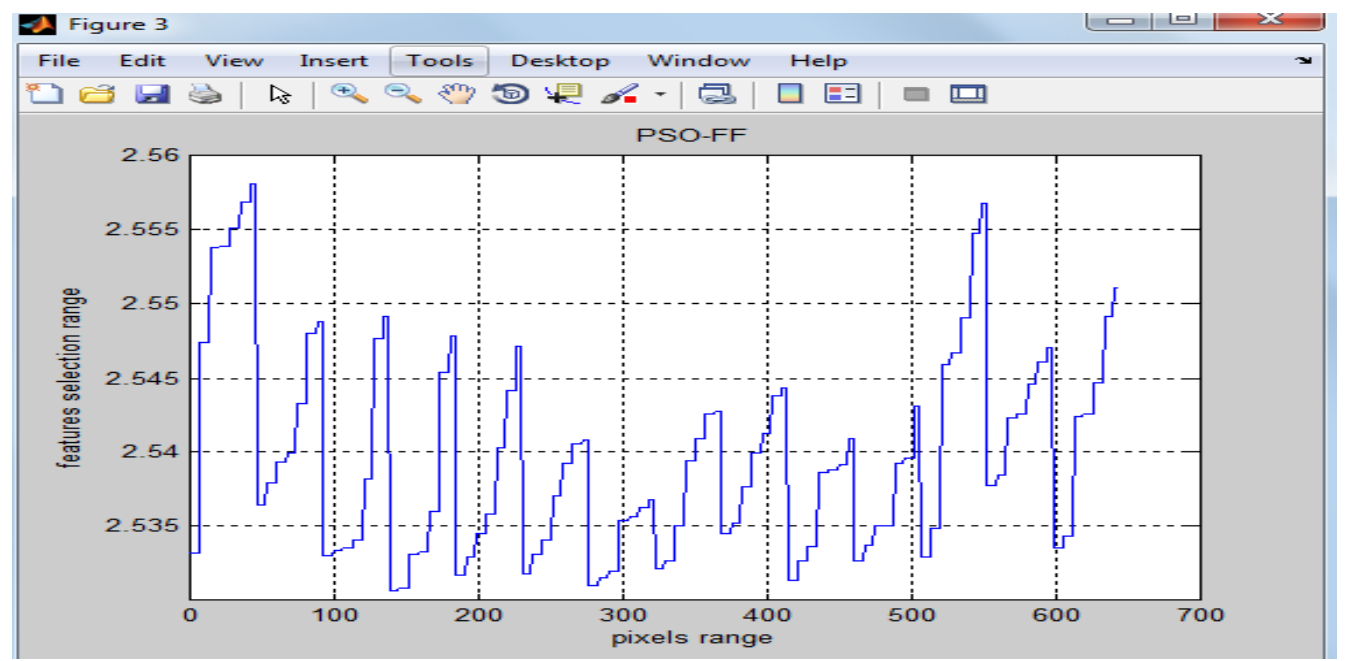

Figure 11:- The range of feature set selected by PSO-FF technique to diagnose the brain disease.

Figure 12 shows the perfect diagnose of Hemochromotosis in computed tomography image. Table $3 \& 4$ give the details of feature extraction through SVD and GLCM techniques.

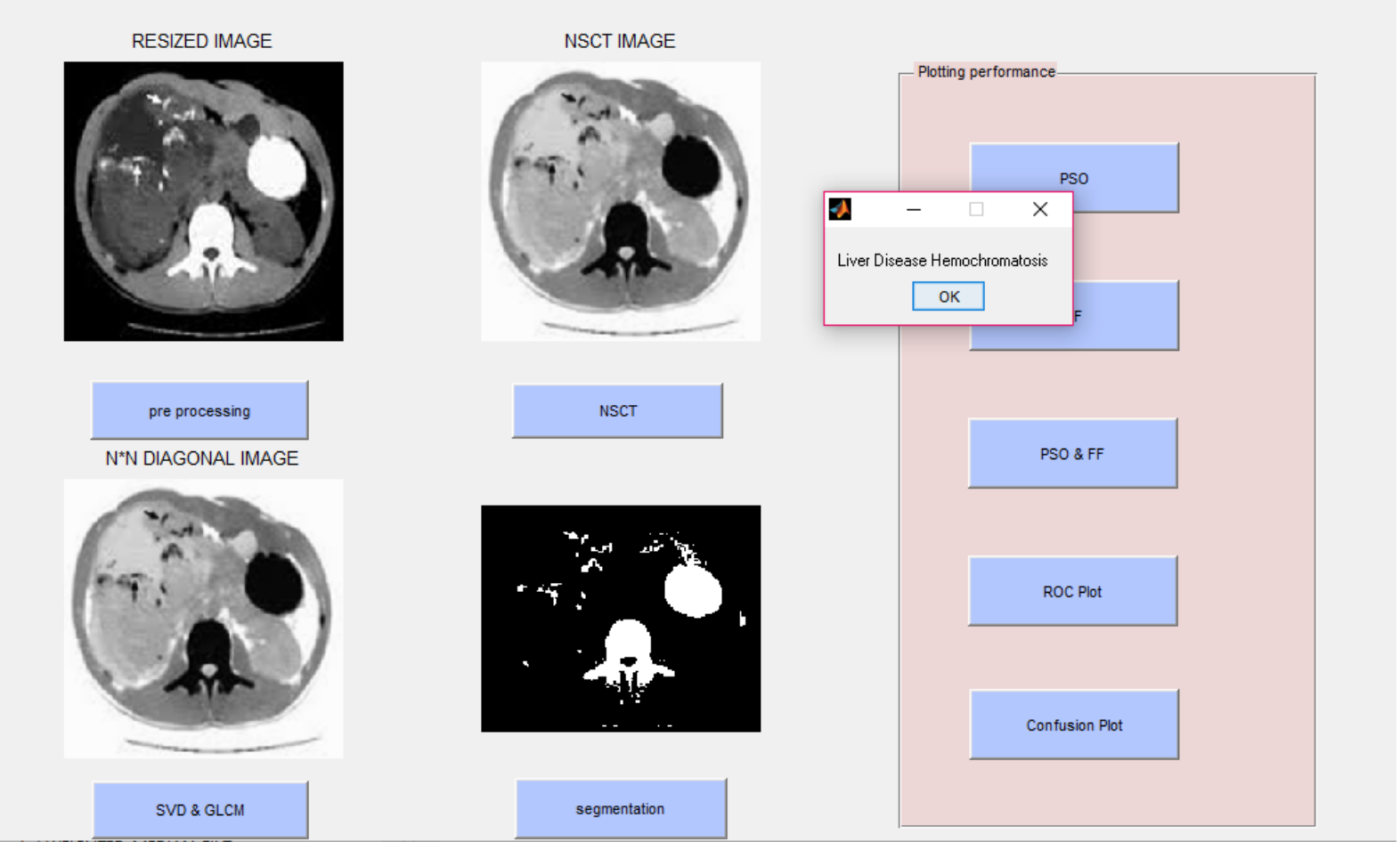

Figure 12:- Perfect prediction of Iron deposit in CT Liver image.

In table 3 the extracted feature values of CT liver for left and right orthogonal matrices and the diagonal matrix through SVD are listed. Table 4 shows the extracted features through GLCM for CT liver image. It has been found from table 4 that the correlation level and the homogeneity are around 0.9 , which is nearer to 1 as required. The contrast level and the energy level must be minimum that are around 0.2. The formulae are given below. 
Table 3:- Feature extraction through SVD method for CT liver image.

\begin{tabular}{|c|c|c|c|}
\hline SVD Features & 'U' matrix & Diagonal matrix $\left(\sum\right)$ & 'V' matrix \\
\hline Mean & 0.0225 & -22.1945 & 0.6301 \\
\hline Standard deviation & 0.0248 & 24.5647 & 0.4265 \\
\hline Min. Pix .Intensity & -0.0053 & -105.1333 & -0.1631 \\
\hline Max. Pix. Intensity & 0.1063 & 5.2444 & 2.3147 \\
\hline
\end{tabular}

Energy $=\sum_{i=0}^{n g-1} \sum_{j=0}^{n g-1} p_{i j}^{2}$

Energy defines the measure of sum of squared elements.

Contrast $=\sum_{n=0}^{n g-1} n^{2} \sum_{i=0}^{n g-1} \sum_{j=0}^{n g-1} p(i . j)$

Contrast measures the intensity variation between the reference pixel and neighbour pixel.

Correlation $=\frac{\sum_{i=0}^{n g-1} \sum_{j=0}^{n g-1}(i, j) p(i, j)-\mu_{x} \mu_{y}}{\sigma_{x} \sigma_{y}}$

Homogeneity $=\sum_{i, j} \frac{p(i, j)}{1+|i-j|}$

Correlation measures, how the reference pixel is related to its neighbour pixel.

Table 4:- Feature extraction through GLCM method for CT liver image.

\begin{tabular}{|c|c|}
\hline GLCM Features & values \\
\hline Contrast & 0.1456 \\
\hline Correlation & 0.9822 \\
\hline Energy & 0.1838 \\
\hline Homogeneity & 0.9377 \\
\hline
\end{tabular}

Figures 13,14,15 describes about the range of features selected by PSO, FF, PSO-FF techniques individually. Fig.13 shows the feature selection range of PSO, the feature selection range is from $0-0.03$ which covers only $50 \%$ of pixels.

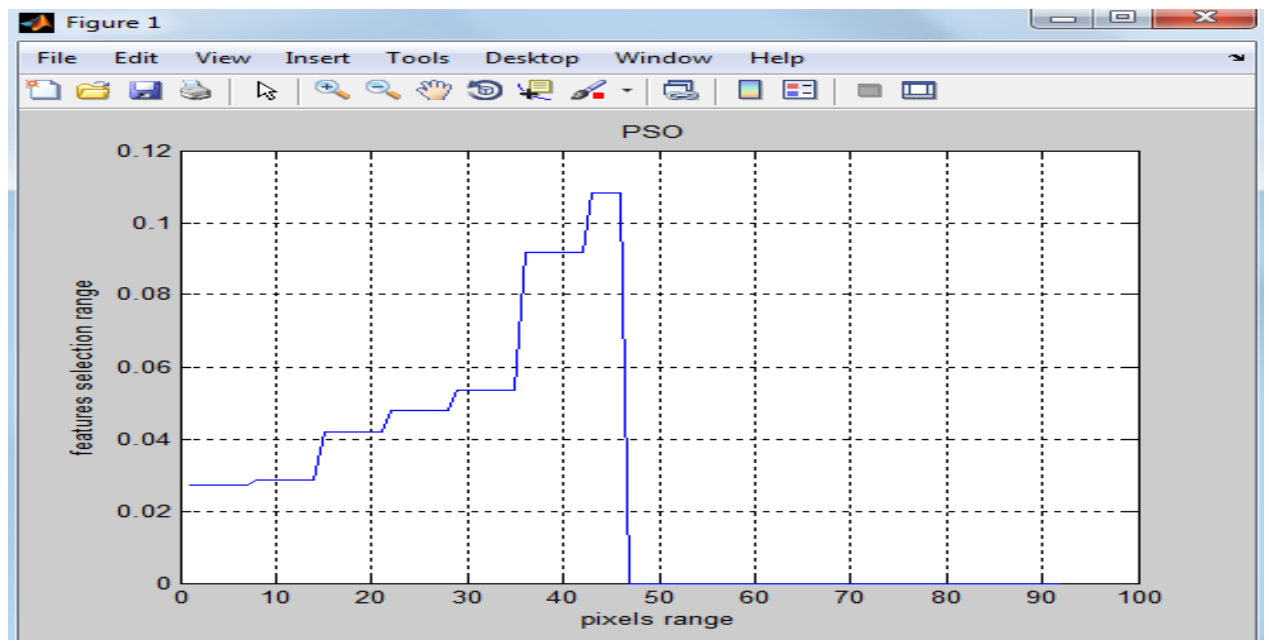

Figure 13:- The range of feature set selected by PSO technique to diagnose the CT liver disease. 
Fig.14 depicts the feature selection range of FF, the feature selection range is from $0-8 \times 10^{13}$ which covers only $10 \%$ of pixels due its fast convergence nature.

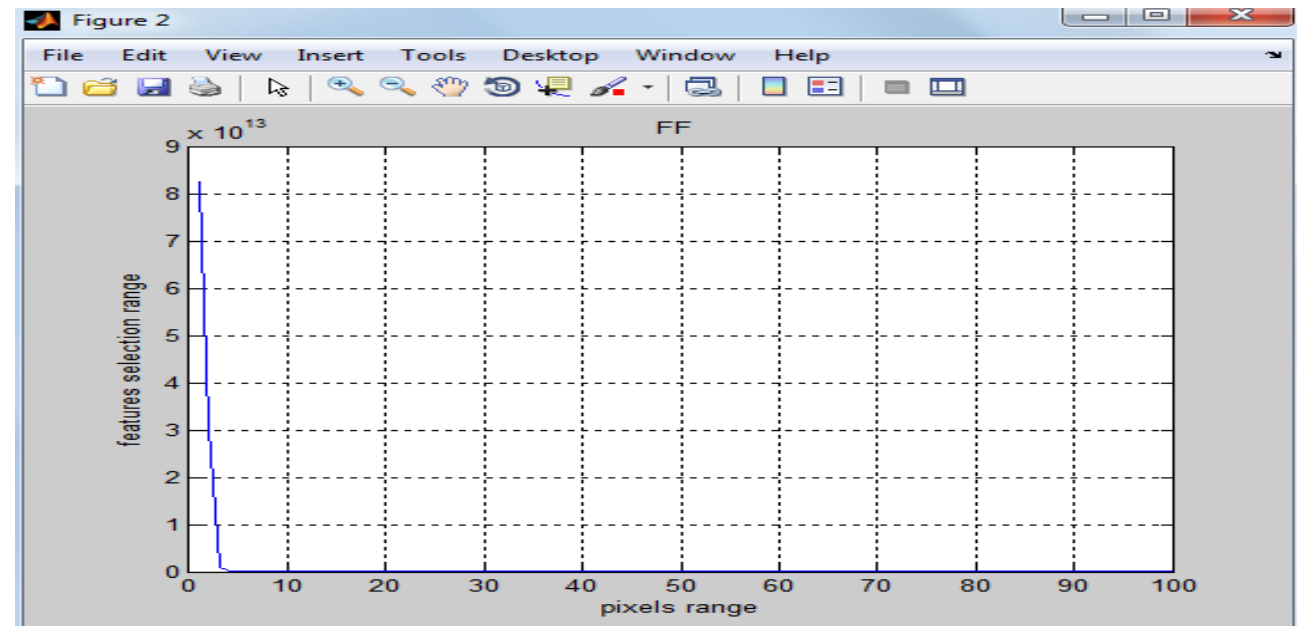

Figure 14:- The range of feature set selected by FF technique to diagnose the CT liver disease.

Fig. 15 describes the feature selection range of PSO - FF, the feature selection range is from 0- 9.9962x $10^{5}$ which covers $75 \%$ of pixels needed for the proposed method .

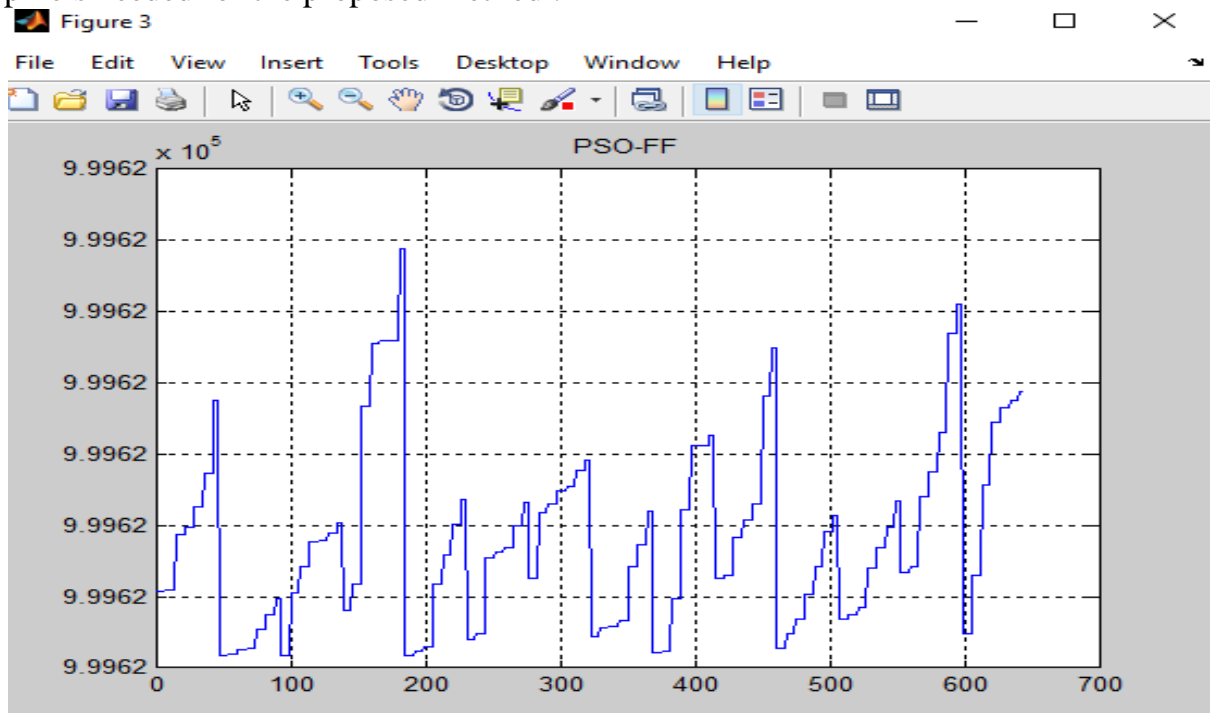

Figure 15:- The range of feature set selected by PSO -FF technique to diagnose the $\mathrm{CT}$ liver disease.

The accuracy of the classifier was evaluated based on the error rate. The error rate was calculated using the terms true positive (TP), true negative (TN), false positive (FP) and false negative (FN). It should be minimized to improve the accuracy of the classifier. Sensitivity and Specificity are the statistical measures used to analyse the performance of the classifier.

True Positive (TP) - Abnormal cases correctly classified.

True Negative (TN) - Normal cases correctly classified.

False Positive (FP) - Normal cases classified as abnormal.

False Negative (FN) - Abnormal cases classified as normal.

Sensitivity represents the capability of the system to diagnose the abnormal cases correctly. Specificity represents the capability of the system to diagnose the normal cases correctly. 


$$
\begin{aligned}
\text { Sensitivity } & =\frac{T P}{T P+F N} \\
\text { Specificity } & =\frac{T N}{T N+F P} \\
\text { Accuracy } & =\frac{T P+T N}{T P+T N+F P+F N}
\end{aligned}
$$

Table 5:- Performance evaluation of classifier for the proposed PSO-FF method.

\begin{tabular}{|c|c|c|c|c|c|c|c|c|}
\hline Sl.no. & $\begin{array}{c}\text { Classification } \\
\text { techniques }\end{array}$ & $\begin{array}{c}\text { True } \\
\text { positive }\end{array}$ & $\begin{array}{c}\text { True } \\
\text { negative }\end{array}$ & $\begin{array}{c}\text { False } \\
\text { positive }\end{array}$ & $\begin{array}{c}\text { False } \\
\text { negative }\end{array}$ & Sensitivity & Specificity & $\begin{array}{c}\text { Accuracy } \\
\text { In } \%\end{array}$ \\
\hline 1. & RBF- SVM & 88 & 119 & 1 & 2 & 0.977 & 0.991 & 98.57 \\
\hline
\end{tabular}

Total no. of normal cases 120. Total no. of abnormal cases 90.

Table 5 interprets the performance analysis of the classifier used. Totally, 210 patients have been taken in to consideration. Among those 120 patients were in normal condition and 90 patients were in abnormal condition. It is seen that the proposed RBF-SVM classifier can able to detect 88 abnormal cases and 119 normal cases correctly with the accuracy of around $99 \%$.

\section{Conclusion:-}

In this paper, the feature selection method based on hybrid meta-heuristics technique has been used for the detection of abnormalities in magnetic resonance brain images and computed tomography liver images. The fitness function for the proposed swarm based firefly optimization technique (PSO-FF) depends upon the minimization of the error rate of the proposed classifier. The performance of the proposed classifier has been studied through the diagnosis of disease through the statistical measures. It has been proved from the analysis that the proposed hybridized PSO-FF technique selects the salient features well with less time and also converges rapidly towards the minimization of the error function by selecting the best possible solutions in the search space, when compared with the individual PSO and FF techniques. The future work will focus on introducing more hybrid meta heuristics based feature selection algorithms to diagnose other types of diseases in MRI brain and CT liver images.

\section{Acknowledgement :-}

The authors are thankful to the Department of Radiology, Raja Muthaiya Medical College and Hospital (RMMCH), Annamalai University for providing us with the required data. The images have also been collected from the data base http//:www.radiopaedia.org.

\section{References:-}

1. Abdenour Mekhmoukh, Karim Mokrani, "Improved Fuzzy C-means based particle Swarm Optimization (PSO) initialization and outlier rejection with level set methods for MR brain image segmentation , "Computer Methods and programs in Bio-medicine, Volume122,Issue 2, Pages 266-281,November 2015.

2. Balasubramanian, VijayaLakshmi, Vasudev Mohan," Kernel-based PSO and FRVM: An automatic plant leaf type detection using texture, shape, and color features", Computers and Electronics in Agriculture, Volume 125, Pages 99-112, July 2016.

3. Esmael Hamuda, Martin Glavin, Edward Jones," A survey of image processing techniques for plant extraction and segmentation in the field", Computers and Electronics in Agriculture, Volume 125, Pages 184-199, July 2016.

4. A.N. Benaichouche, H.Oulhadj, P.Siarry, "improved spatial fuzzy c-means clustering for image segmentation using pso initialization, mahalanobis distance and post-segmentation correction", Digital signal processing, volume 23, issue 5,pages 1390-1400, September 2013.

5. Yong Zhang, Dan Huang, Min Ji, Fuding Xie, "Image segmentation using PSO and PCM with Mahalanobis distance", Expert Systems with Applications, Volume 38, Issue 7, Pages 9036-9040, July 2011.

6. Devraj Mandal, Amitava Chatterjee', Madhubanti Maitra,"Robust medical image segmentation using particle swarm optimization aided level set based global fitting energy active contour approach,"Engineering Applications of Artificial Intelligence, Volume 35, Pages 199-214, October 2014. 
7. Pablo Mesejo, Oscar Ibanez, Oscar Cordon, Stefano Cagnoni, "A survey on image segmentation using metaheuristics based deformable models: State of the art and critical analysis, "Applied Soft Computing, volume 44, pages 1-29,July 2016.

8. Jothi G, Hannah Inbarani H, " Hybrid tolerance rough set-firefly based supervised feature selection for mri brain tumor image classification, "Applied Soft computing volume 46, pages 639-651, September 2016.

9. V. Rajinikanth, M.S Couceiro, "RGB histogram based color image segmentation using firefly algorithm," Procedia Computer Science, Volume 46,Pages 1449-1457, 2015.

10. Adan Jose-Garcia, Wilfrido Gomez- Flores, "Automatic clustering using nature - inspired metaheuristics: A survey”, Applied Soft Computing, Volume 41, Pages 192-213, April 2016.

11. Satyasai Jagannath Nanda, Ganapati Panda, "A survey on nature inspired metaheuristic algorithms for partitional clustering, Swarm and Evolutionary Computation, Volume 16, Pages 1-18, June 2014.

12. Xin-She Yang, "Firefly Algorithms", Nature-Inspired Optimization Algorithms, Chapter 8, Pages 111-127, 2014.

13. Iztok Fister, Iztok Fister jr., Xin- She Yang, Janez Brest, "A comprehensive review of firefly algorithms", Swarm and Evolutionary computation, Volume 13, Pages 34-46, December 2013.

14. Nguyen Cong Long, Phayung Meesad, Herwig Unger, "A highly accurate firefly based algorithm for heart disease prediction," Expert Systems with Applications, Volume 42, Issue 21,Pages 8221-8231, November 2015.

15. Mohammed Alweshah, Salwani Abdullah, "Hybridizing firefly algorithm with a probabilistic neural network for solving classification problems", Applied Soft Computing, Volume 35, Pages 513-524, October 2015.

16. Yong Zhang, Dunwei Gong, Ying Hu, Wanqiu Zhang, "Feature selection algorithm based on bare bones particle swarm optimization", Neurocomputing, Volume 148, Pages 150-157,January 2015.

17. Sandeep Chaplot, L.M.Patnaik, N.R.Jagannathan, "Classification of magnetic resonance brain images using wavelets as input to support vector machine and neural network", Biomedical signal processing and control, vol 1, pages 86-92, 2006.

18. Fahd M.A.mohsen, Mohiy M.Hadhoud, Khalid Amin, “A new optimization -based image segmentation method by Particle Swarm Optimization", International Journal of Advanced Computer Science and Applications, Special issue on image processing and Analysis, pages 10-18.

19. H.Hannah Inbarani, Ahmad Taher Azar, G. Jothi," Supervized hybrid feature selection based on PSO and rough sets for medical diagnosis", Computer methods and programs in bio-medicine, 113,pages 175-185,2014.

20. Bae- Muu Chang, Hung- Hsu Tsai, Chih- Yuan Yen, "SVM - PSO based rotation - invariant image texture classification in SVD and DWT domains", Engineeering Applications of Artificial Intelligence, 52, pages 96107, 2016.

21. Nelly Gordillo, Eduard Montseny, Pilar Sobrevilla, "State of the art Survey on MRI brain tumor segmentation ", Magnetic resonance imaging , 31, pages 1426-1438, 2013.

22. Amita Kumari, Rajesh Mehra,"Hybridized Classification of Brain MRI using PSO \& SVM", International Journal of Engineering and Advanced Technology (IJEAT), Volume-3, Issue-4, pages 319-323. April 2014.

23. S.Anu H. Nair', P. Aruna, "Comparison of DCT, SVD and BFOA based multimodal biometric watermarking systems", Alexandria Engineering Journal, Volume 54, Issue 4, Pages1161-1174, December 2015.

24. Arthur L. da Cunha, Jianping Zhou, Minh N. Do, “ The Non- sub sampled Countourlet Transform: Theory, Design and Applications", IEEE Transactions on image processing, Volume 15, no.10, Pages -3089-3101, 2006.

25. He Xiaolan, Wu Yili, “ Texture Feature Extraction Method Combining Non- sub sampled Contour Transformation with Gray Level Co-occurrence Matrix", Journal of multimedia, vol. 8, no. 6, pages 675-684, 2013.

26. Kurakula Sravya , Dr. P. Govardhan, Naresh Goud M, "Image Fusion on Multi Focused Images using NSCT", International Journal of Computer Science and Information Technologies, Vol. 5 (4) , 5393-5396, 2014.

27. Kirk Baker, "Singular Value Decomposition Tutorial", March 29, pages 1-24, 2005 (Revised January 14, 2013),

28. Haralick R M,Shanmugam K, Dinstein L, “ Textural features for image lassification”, IEEE Transactions on Systems, Man and Cybernetics, 3(6), pages 610-621, 1973.

29. P.Mohanaiah, P.Sathyanarayana, L.GuruKumar, "Image Texture Feature Extraction Using GLCM Approach," International Journal of Scientific and Research Publications, Volume 3, Issue 5, Pages 1-5, May 2013. 
30. Punal M.Arabi, Gayatri Joshi, N. Vamsha Deepa, "Performance evaluation of GLCM and pixel intensity matrix for skin texture analysis, "Perspectives in Science, Available online- In Press, Corrected proof - Note to users, April 2016.

31. Lahouaoui Lalaoui, Tayeb Mohamadi, Abdelhak Diaalab, "New method for image segmentation," Procedia Social and Behavioral Sciences, Volume 195, Pages 1971-1980, July 2015.

32. M.N.V.S.S. Kumar,G. Sasi Bhushana Rao, L.Ganesh, Rajkumar Goswami, “ Expectation - Maximization based image fusion algorithm for Detection of under water targets from SONAR images, " Procedia Computer Science, International Conference on Computational Modelling and Security, Volume 85,Pages 782-789, 2016. 